\title{
Twenty-five years of applied mathematical programming and modelling
}

\author{
Christina Erlwein-Sayer ${ }^{1}$ • Ronald Hochreiter $^{2}$ (D)
}

Published online: 12 June 2018

(C) Springer-Verlag GmbH Germany, part of Springer Nature 2018

\section{Preface}

In 1991 Gautam Mitra started the conference series APMOD, i.e. applied mathematical programming and modelling. The initial conference was held at Brunel University in West London and was re-iterated every 2 or 3 years in various European cities such as Budapest (1993), London (1995), Limassol (1998), London (2000), Varenna (2002), London (2004), Madrid (2006), Bratislava (2008), Paderborn (2012), and Warwick (2014).

Twenty-five years later, one quarter of a century after this successful conference series has been initiated, it was held in Brno, Czech Republic in June 2016. The conference has been organized in collaboration with Brno University of Technology and this special anniversary was celebrated with a laudation for Prof. Gautam Mitra during the opening session of the conference.

Contributions presented at the conference ranged from optimization modelling frameworks, problem solution methods and software to various applications of optimization. Furthermore, contributions from the field of Data Science as well as Business Analytics especially in relation to optimization were included. Relevant application areas comprise of but are not restricted to Finance, Transportation, Supply Chain Planning and Management, Revenue Management, Energy and Infrastructure. Decision models may be deterministic or stochastic and the methodological focus of the con-

$凶$ Ronald Hochreiter

ronald.hochreiter@wu.ac.at

Christina Erlwein-Sayer

christina@optirisk-systems.com

1 OptiRisk Systems, 1 One Oxford Rd, Denham, Uxbridge UB9 4DA, UK

2 WU Vienna University of Economics and Business, Welthandelsplatz 1, 1020 Vienna, Austria 
ference has been gradually extended from pure Mathematical Programming including Stochastic Optimization to Heuristics, Metaheuristics and Simulation.

APMOD 2016 comprised 55 talks and three plenaries from internationally renowned experts. In this volume six outstanding research papers mainly from the field of Finance were selected from these contributions, and will be introduced below. A further paper related to Finance submitted to CMS without targetting a special issue completes the present collection.

An additional conference proceedings issue (Hochreiter 2017) which has been published in the journal ITM Web of Conferences is available and depicts the broad range of topics the conference series covers, i.e. containing papers from the fields of engineering, computer science and machine learning as well as political science.

In the first paper of this special issue entitled Portfolio Selection under Supply Chain Predictability Thomas Trier Bjerring, Kourosh Marjani Rasmussen and Alex Weissensteiner investigate whether returns of industry portfolios can be used to predict returns of other industry portfolios. They find a lead-lag structure that suggests that information diffuses only gradually across industries. Furthermore, this information is used in a portfolio optimization context and shows attractive results for investment managers.

Oleksandra Putyatina and Jörn Sass consider a portfolio optimization problem by maximizing expected utility of terminal wealth in a continuous-time financial market. In their paper Approximation for Portfolio Optimization in a Financial Market with Shot-Noise Jumps stock prices are driven by a Brownian motion as well as a shot-noise process, whereby this shot-noise part leads to jumps in the stock prices whose influence decays exponentially with time. As explicit solutions are difficult, two approximation schemes are shown and compared within simulation studies to obtain promising results.

The paper ALM models based on Second Order Stochastic Dominance by Maram Alwohaibi and Diana Roman considers an Asset and Liability Management (ALM) case in which the risk of underfunding is modeled based on the concept of stochastic dominance. The resulting model is successfully applied to a financial planning problem of a defined benefit pension fund in Saudi Arabia.

The author Yeliz Yolcu-Okur and her colleagues Tilman Sayer, Bilgi Yilmaz and B. Alper Inkaya employ Malliavin calculus techniques to compute the Delta of European type options in the presence of stochastic volatility to obtain a general formula for the Malliavin weight and apply the derived formula to the well-known models of SteinStein and Heston in the paper Computation of the Delta of European Options under Stochastic Volatility Models.

Abdulwahab Animoku focusses on the reconstruction of volatility surfaces via a Bayesian framework in the paper Modeling and Implementation of Local Volatility Surfaces in Bayesian Frameworks with her colleagues Yeliz Yolcu-Okur and Omur Ugur. In this paper the (stochastically) parametrized volatility structure in Dupire local volatility mode is used to develop and implement novel mathematical tools for handling the classical methods of constructing local volatility surfaces.

Heng Xiong and Rogemar Mamon do what the title of their paper Putting a price tag on temperature promises. A model for the evolution of daily average temperatures is developed to support the analysis of weather derivatives. To do so an Ornstein- 
Uhlenbeck (OU) process modulated by a hidden Markov chain (HMM) is proposed to model both the mean-reversion and stochasticity of a de-seasonalised component. The results are tested using a 4-year Toronto temperature data set and a sensitivity analysis of the option prices with respect to model parameters has been conducted.

Finally, Taras Bodnar, Yarema Okhrin, Valdemar Vitlinskyy, and Taras Zabolotskyy study exponential and quadratic utility functions often used in portfolio allocation and link the resulting portfolios maximizing utility to the corresponding optimal portfolios on the minimum Value-at-Risk (VaR) approach. Analytic expressions for the risk aversion coefficients as functions of the VaR level are obtained if the underlying distribution is elliptically contoured. Assuming parameter uncertainty, confidence intervals for the risk coefficients are derived and validated in an empirical study.

Acknowledgements We are grateful to Prof. Rüdiger Schultz, the Editor-in-Chief of the journal Computational Management Science to enable the publication of this special issue in this prestigious journal.

\section{Reference}

Hochreiter R (2017) Applied mathematical programming and modelling 2016. ITM Web Conf 14:00001. https://doi.org/10.1051/itmconf/20171400001 